\title{
Pinning Control of Spatiotemporal Chaos
}

\author{
R. O. Grigoriev, M. C. Cross, and H. G. Schuster \\ Condensed Matter Physics 114-36 and Neural Systems Program 139-74, California Institute of Technology, \\ Pasadena, California 91125
}

(Received 29 April 1997)

\begin{abstract}
Linear control theory is used to develop an improved localized control scheme for spatially extended chaotic systems, which is applied to a coupled map lattice as an example. The optimal arrangement of the control sites is shown to depend on the symmetry properties of the system, while their minimal density depends on the strength of noise in the system. The method is shown to work in any region of parameter space and requires a significantly smaller number of controllers compared to the method proposed earlier by $\mathrm{Hu}$ and $\mathrm{Qu}$ [Phys. Rev. Lett. 72, 68 (1994)]. A nonlinear generalization of the method for a 1D lattice is also presented. [S0031-9007(97)04237-3]
\end{abstract}

PACS numbers: 05.45. $+\mathrm{b}, 02.30 . \mathrm{Wd}$

Controlling chaos in high-dimensional systems [1] and spatiotemporal chaos especially is a very important problem with numerous applications to turbulence [2], instabilities in plasma [3], multimode lasers [4], and reaction-diffusion systems [5].

The present Letter represents an effort to develop a general control algorithm for spatiotemporally chaotic systems using the methodology of linear control theory, which already proved to be fruitful [6]. Clarifying a number of issues will have direct bearing on this. For instance, it is not clear how many parameters are required for successful control. If the control is applied locally, what is the minimal density of controllers and how should they be arranged to obtain optimal performance? What are the limitations of the linear control scheme and how can they be overcome?

Consider the coupled map lattice (CML), originally introduced by Kaneko [7], in an alternative form:

$$
\begin{aligned}
z_{i}^{t+1} & =F\left(z_{i-1}^{t}, z_{i}^{t}, z_{i+1}^{t}\right) \\
& =f\left((1-2 \epsilon) z_{i}^{t}+\epsilon\left(z_{i-1}^{t}+z_{i+1}^{t}\right)\right),
\end{aligned}
$$

with $i=1,2, \cdots, L$ and periodic boundary conditions (BC), $z_{i+L}^{t}=z_{i}^{t}$ imposed. We also assume that the local map $f(z, a)$ is a nonlinear function with parameter $a$, such that $f\left(z^{*}, a\right)=z^{*}$.

To be specific, we choose

$$
f(z)=a z(1-z),
$$

but emphasize that all the major results hold independent of this choice. This CML has a homogeneous steady state $z^{*}=1-1 / a$, which is unstable for $a>3.0$. Our goal is to stabilize it using a minimal number of controllers.

The first attempt in this direction was undertaken by $\mathrm{Hu}$ and $\mathrm{Qu}$ [8]. The authors tried to stabilize the homo- geneous state by controlling an array of $M$ periodically placed pinning sites $\left\{i_{1}, \cdots, i_{M}\right\}$ with appropriately chosen control $u_{m}^{t}$

$$
z_{i}^{t+1}=F\left(z_{i-1}^{t}, z_{i}^{t}, z_{i+1}^{t}\right)+\sum_{m=1}^{M} \delta\left(i-i_{m}\right) u_{m}^{t} .
$$

This however required a very dense array with distance between controllers $L_{p}=L / M \leq 3$ in the physically interesting interval of parameters $3.57<a<4.0$.

The reason for this is the spatial periodicity of the pinnings. Since the system is spatially uniform, its eigenmodes are just Fourier modes and the control does not affect the modes whose nodes happen to lie at the pinnings, i.e., modes with periods equal to $2 L_{p}, 2 L_{p} / 2,2 L_{p} / 3$, etc., provided those are integer. The control scheme worked only when all such modes were stable.

It is however not necessary to destroy the periodicity completely to achieve control: that would complicate the analysis unnecessarily. Instead we add one more pinning site between each of the existing ones. Not all positions are good, but some do solve the problem-previously uncontrollable modes become controllable.

In order to understand how the pinnings should be placed and see whether we achieve improved performance by introducing additional controllers, we have to use a few results of the linear control theory [9]. We will start with linearizing Eq. (3) about the homogeneous steady state $\mathbf{z}^{t}=\left(z^{*}, \cdots, z^{*}\right)$ in both the state vector and control to obtain the following standard equation

$$
\mathbf{x}^{t+1}=A \mathbf{x}^{t}+B \mathbf{u}^{t},
$$

where we denoted the displacement $\mathbf{x}=\mathbf{z}-\mathbf{z}^{*}$. If we define $\alpha=\partial f\left(x^{*}, a\right) / \partial x$, then the $L \times L$ Jacobian $A$ is given by

$$
A=\alpha\left(\begin{array}{ccccc}
1-2 \epsilon & \epsilon & 0 & \cdots & \epsilon \\
\epsilon & 1-2 \epsilon & \epsilon & \cdots & 0 \\
0 & \epsilon & 1-2 \epsilon & \cdots & 0 \\
\vdots & \vdots & \vdots & \ddots & \vdots \\
\epsilon & 0 & 0 & \cdots & 1-2 \epsilon
\end{array}\right)
$$


and the $L \times M$ control matrix $B_{i j}=\sum_{m} \delta(j-$ $m) \delta\left(i-i_{m}\right)$ depends on how we place the pinning sites.

If we use synchronous linear feedback $\mathbf{u}^{t}=-K \mathbf{x}^{t}$, Eq. (4) becomes

$$
\mathbf{x}^{t+1}=(A-B K) \mathbf{x}^{t},
$$

and the solution $\mathbf{x}=0$ can be made stable by a suitable choice of the feedback gain matrix $K$, if the controllability condition rank $(C)=L$ is satisfied. The controllability matrix $C$ is defined via

$$
C=\left(B A B \cdots A^{L-1} B\right) .
$$

One can easily verify that the matrix $B$ calculated for a periodic array of pinning sites does not satisfy the controllability condition and therefore the homogeneous steady state is not controllable. It can be stabilized if the weaker stabilizability condition is satisfied, i.e., all uncontrollable modes are stable. However this imposes excessive restrictions on the pinning density.

The condition for stabilizability can be obtained from the spectrum of eigenvalues of the matrix (5)

$$
\gamma_{i}=\alpha\left[1-2 \epsilon\left(1-\cos \left(k_{i}\right)\right],\right.
$$

where $k_{1}=0, k_{i}=k_{i+1}=\pi i / L$ for $i=2,4,6, \ldots$ and, for $L$ even, $K_{L}=\pi$ and $\alpha=2-a$. Specifically, we need $\left|(a-2)\left[1-2 \epsilon\left(1-\cos \left(\pi j / L_{p}\right)\right)\right]\right|<1$ for all $j=1, \ldots, L-2$, such that $L_{p} / j$ is integer. Using this criterion one can obtain the relation between the minimum coupling, the distance between controllers and parameter $a$ of the local chaotic map for a stabilizable system. For instance, $j=1$ yields

$$
\epsilon=\frac{a-3}{2(a-2)\left[1-\cos \left(\pi / L_{p}\right)\right]} .
$$

The numerically obtained results of $\mathrm{Hu}$ and $\mathrm{Qu}$ (Fig. 1) are seen to lie exactly on the curves, given by Eq. (9).

It is possible however to extend the limits of the control scheme quite substantially by making the system controllable as opposed to stabilizable. This is easily achieved by choosing a different matrix $B$, i.e., placing the pinning

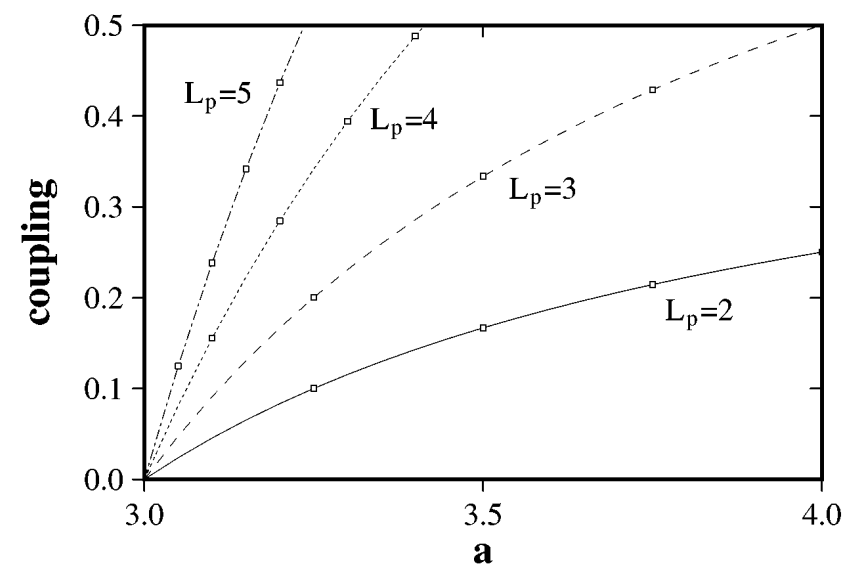

FIG. 1. Periodic array of single pinning sites: Critical coupling $\epsilon_{c r}$ as a function of parameter $a$. The data points represent the numerical results from Fig. 2 of [8], with $\epsilon$ rescaled by a factor of 2 to make it compatible with our definition. sites differently. Doing so will enable us to control the system anywhere in the parameter space at the same time using a smaller density of controllers.

First one has to determine the dimensionality of the matrix $B$, in other words determine the minimal number of parameters required to control the CML (1) of an arbitrary length. It can be shown [10] that the minimal number of parameters required to control a system with degenerate Jacobian is equal to the greatest multiplicity of its eigenvalues.

Since the system considered has parity symmetry, the eigenvalues (8) of its Jacobian are in fact doubly degenerate, so the minimal number of control parameters yielding a controllable system in our case is two, meaning at least two pinning sites are required. One can easily verify that the controllability condition for an $L \times 2$ matrix

$$
B_{i j}=\delta(j-1) \delta\left(i-i_{1}\right)+\delta(j-2) \delta\left(i-i_{2}\right)
$$

is indeed satisfied for a number of arrangements $\left\{i_{1}, i_{2}\right\}$. The restrictions on the mutual arrangement of the controllers are again given by the condition of controllability: $L$ should not be a multiple of $\left|i_{2}-i_{1}\right|$; otherwise the mode with the period $2\left|i_{2}-i_{1}\right|$ becomes uncontrollable.

The next step in the algorithm is to determine the feedback gain $K$. Pole placement techniques based on Ackermann's method [11] are inapplicable to the problem of controlling spatially extended systems because they are numerically unstable [12] and break down rapidly for problems of order greater than 10.

Instead we use the method of the linear-quadratic (LQ) control theory [9], applicable to the unstable periodic trajectories as well as fixed points. This method is not only numerically stable, but also allows one to optimize the control algorithm to increase convergence speed, and at the same time minimize the strength of control. As we will see below, decreasing control enlarges the basin of attraction, which has very important consequences for the time to achieve control (capture the chaotic trajectory). The optimal solution is obtained by minimizing the cost functional

$$
V\left(\mathbf{x}^{0}\right)=\sum_{t=0}^{\infty}\left(\mathbf{x}^{t \dagger} Q \mathbf{x}^{t}+\mathbf{u}^{t \dagger} R \mathbf{u}^{t}\right)
$$

where $Q$ and $R$ are the weight matrices that can be chosen as any positive-definite square matrices.

The minimum of (11) is reached when

$$
K=\left(R+B^{\dagger} P B\right)^{-1} B^{\dagger} P A,
$$

where $P$ is the solution to the discrete-time algebraic Ricatti equation

$$
P=\left(Q+A^{\dagger} P A\right)-A^{\dagger} P^{\dagger} B\left(R+B^{\dagger} P B\right)^{-1} B^{\dagger} P A .
$$

Numerical simulations show that the CML (1), (2) can indeed be stabilized by this linear control scheme in a wide range of parameters $a$ and $\epsilon$. The solution for $K$ is presented in Fig. 2 for $a=4.0, \epsilon=0.33$, and $L=8$ with 


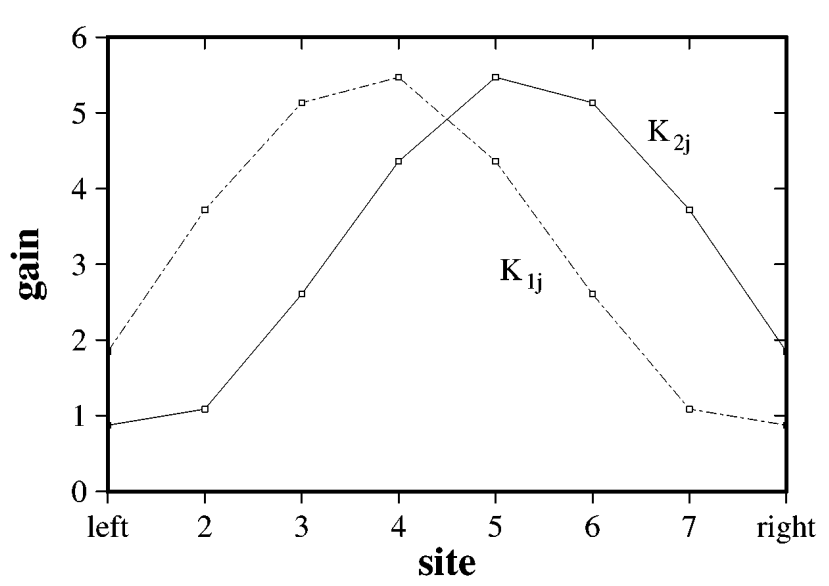

FIG. 2. Feedback matrix: Feedback gain vectors $K_{1 j}$ and $K_{2 j}$ for left and right controllers placed at the sides of the lattice $\left(i_{1}=1, i_{2}=8\right)$ as functions of the lattice site for $a=4.0$ and $\epsilon=0.33$.

$Q=I_{8 \times 8}$ and $R=I_{2 \times 2}$. The steady homogeneous state $z^{*}=0.75$ has three unstable and five stable directions and we use two pinning sites to control it.

The contribution $-K_{m i} x_{i}^{t}$ from the site $i$ far away from the pinning site $i_{m}$ is larger, as expected: since the feedback is applied indirectly through coupling to the neighbors, the perturbation introduced by the controllers decays with increasing distance to the pinning sites.

Noise limits our ability to locally control arbitrarily large systems with local interactions. We estimate the largest length of the system using the controllability condition, which in our case determines whether there exists control $\mathbf{u}^{t}, \ldots, \mathbf{u}^{t+L-1}$, bringing an arbitrary initial state $\mathbf{x}^{t}$ to an arbitrary final state $\mathbf{x}^{t+L}$. Without noise

$$
\mathbf{x}^{t+L}=A^{k} \mathbf{x}^{t}+\sum_{\tau=0}^{L-1} \sum_{m=1}^{2} A^{L-1-\tau} \mathbf{b}^{m} u_{m}^{t+\tau},
$$

where $\mathbf{b}^{m}$ is the $m$ th column of $B$.

However, if there is noise of relative magnitude $\sigma$ at time $t$, it will be amplified by a factor of $\gamma$ per iteration, where $|\gamma|=\exp \left(\lambda_{\max }\right)$ is the largest eigenvalue (8) of the Jacobian. At time $t+L$ its magnitude will be $\sigma|\gamma|^{L}$. For the control to work, all terms $A^{L-1-\tau} \mathbf{b}^{i} u_{i}^{t+\tau}$ should be of the same order as or exceed the amplified noise.

Indeed, the perturbation $\delta x_{i_{m}}^{\tau}$ introduced by the controller $i_{m}$ affects the dynamics of the remote site $j$ only after propagating a distance $\Delta=\left|i_{m}-j\right|$ in time $T=\Delta$, decaying by a factor of $\alpha \epsilon$ per iteration. Consequently the dynamics of site $j$ at time $t+L$ will be affected by control applied only at times $t, \ldots, t+L-T-1$. This dictates $O\left(\delta x_{i_{m}}^{t+L-T-1}(\alpha \epsilon)^{T}\right) \gtrsim \sigma|\gamma|^{L}$.

Because of the periodic BC $0 \leq \Delta \leq L / 2$. On the other hand, $\left|\delta x_{i}^{\tau}\right|<\delta x \sim 1$, where $\delta x$ determines the range, where the linear approximation (4) is valid. As a result we obtain the estimates on the size of the controllable system, similar to those obtained by Aranson et al. for the lattice with asymmetric coupling [cf. Eq. (15) of
Ref. [13]]. The tightest bounds are given by $\Delta=0$ :

$$
L_{\max }^{(1)}=-\frac{\ln (\sigma)}{\lambda_{\max }}, \quad 0.5<\epsilon<1,
$$

and $\Delta=L / 2$ :

$$
L_{\max }^{(2)}=\frac{2 \ln (\sigma)}{\ln (\epsilon)-\lambda_{\max }}, \quad 0<\epsilon<0.5 .
$$

The maximal length of the system that can actually be stabilized by the LQ method with two pinning sites, placed next to each other, is obtained numerically by choosing the fixed point as the initial condition and monitoring the evolution of the system in the presence of noise under control (12), calculated for $Q=I_{L \times L}$ and $R=I_{2 \times 2}$. This length is quite large for the moderate level of noise (Fig. 3) and is rather close to the values (15) and (16) where the controllability breaks down.

The problem of controlling a large one-dimensional system with the length $L>L_{\max }(\sigma)$ can be easily reduced to the problem of independently controlling a number of smaller systems with the length $L_{p}<L_{\max }(\sigma)$. We partition the entire lattice $\left\{z_{1}^{t}, \ldots, z_{L}^{t}\right\}$ into $M=L / L_{p}$ subdomains $\left\{z_{(m-1) L_{p}+1}^{t}, \ldots, z_{m L_{p}}^{t}\right\}$, and control it with an array of pinning sites $i_{m 1}=(m-1) L_{p}+1, i_{m 2}=m L_{p}$, $m=1, \ldots, M$, positioned periodically at the boundaries of these subdomains.

The stabilization can be achieved by choosing

$$
\begin{aligned}
u_{i_{m 1}}^{t}= & F\left(z_{i_{m 2}}^{t}, z_{i_{m 1}}^{t}, z_{i_{m 1}+1}^{t}\right)-F\left(z_{i_{m 1}-1}^{t}, z_{i_{m 1}}^{t}, z_{i_{m 1}+1}^{t}\right) \\
& +\prod_{i=1}^{L_{p}} \theta\left(\delta x_{i}-\left|x_{(m-1) L_{p}+i}^{t}\right|\right) \sum_{i=1}^{L_{p}} K_{1 i} x_{(m-1) L_{p}+i}^{t} \\
u_{i_{m 2}}^{t}= & F\left(z_{i_{m 2}-1}^{t}, z_{i_{m 2}}^{t}, z_{i_{m 1}}^{t}\right)-F\left(z_{i_{m 2}-1}^{t}, z_{i_{m 2}}^{t}, z_{i_{m 2}+1}^{t}\right) \\
& +\prod_{i=1}^{L_{p}} \theta\left(\delta x_{i}-\left|x_{(m-1) L_{p}+i}^{t}\right|\right) \sum_{i=1}^{L_{p}} K_{2 i} x_{(m-1) L_{p}+i}^{t},
\end{aligned}
$$

where $\theta(x)$ is a step function.

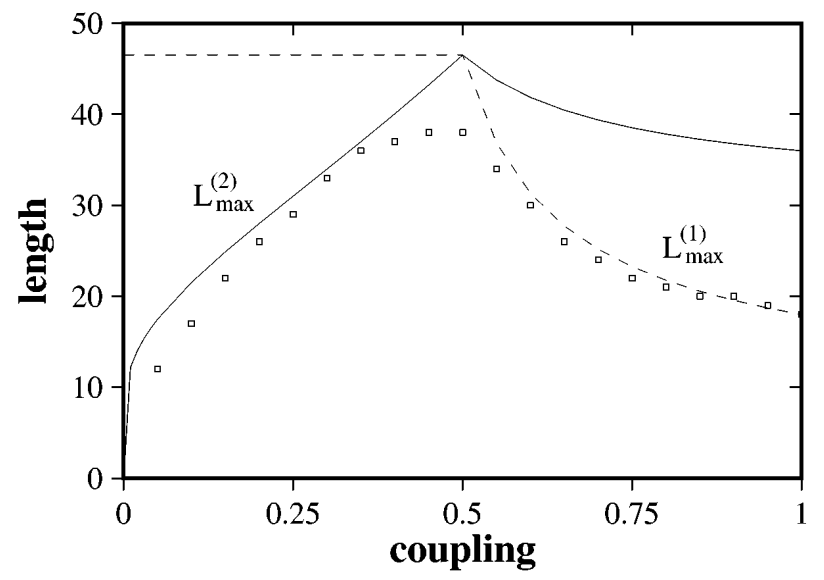

FIG. 3. The largest length of the lattice which can be controlled with two pinning sites: Theoretical estimates (solid lines) and numerical results (dots) obtained with the uniformly distributed noise of amplitude $\sigma=10^{-14}$ as functions of coupling $\epsilon$ for $a=4.0$. 
This arrangement effectively carries two functions. We use control (17) to (nonlinearly) decouple the subdomains, simultaneously imposing periodic boundary condition for each subdomain (the first two terms) to make the system controllable. Then we stabilize each subdomain asynchronously by applying a linear (in deviation $x_{i}^{t}=z_{i}^{t}-z^{*}$ ) feedback (the last term), inside the neighborhood of the fixed point determined by $\delta x_{i}$. Since the linear approximation (4) is valid only if

$$
\delta x_{i} \ll\left|K_{m i}\right|^{-1}, \quad m=1, \ldots, M,
$$

strong feedback significantly decreases the size of the capture region, which makes the capture time large. Reduction of the capture time can be achieved by minimizing the feedback strength using the LQ method (12), (13).

We demonstrate this approach by stabilizing the homogeneous stationary state of the CML defined by Eqs. (1) and (2) with $a=4.0, \epsilon=0.33 . L=128$ sites were divided into $M=16$ subdomains of length $L_{p}=8$, each controlled by two pinning sites. The results presented in Fig. 4 show the evolution of the system from the initial condition chosen to be a collection of random numbers in the interval $[0,1]$.

Equations (15) and (16) now give the minimal density of pinning sites that yields the controllable fixed point solution. It is indeed seen to be much lower than that given by (9), e.g., $2 / L_{p}=1 / 20$ (2/37 from the numerics; see Fig. 3) as opposed to $1 / L_{p}=1 / 2$ for the choice $a=$ 4.0, $\epsilon=0.4$ and the precision of calculations given by $\sigma=10^{-14}$.

Although the resulting control scheme becomes nonlinear (and therefore requires full knowledge of the evolution equations), it has the additional benefit, that the capture time is determined by the length $L_{p} \ll L$ and is typically many orders of magnitude smaller than that ob-

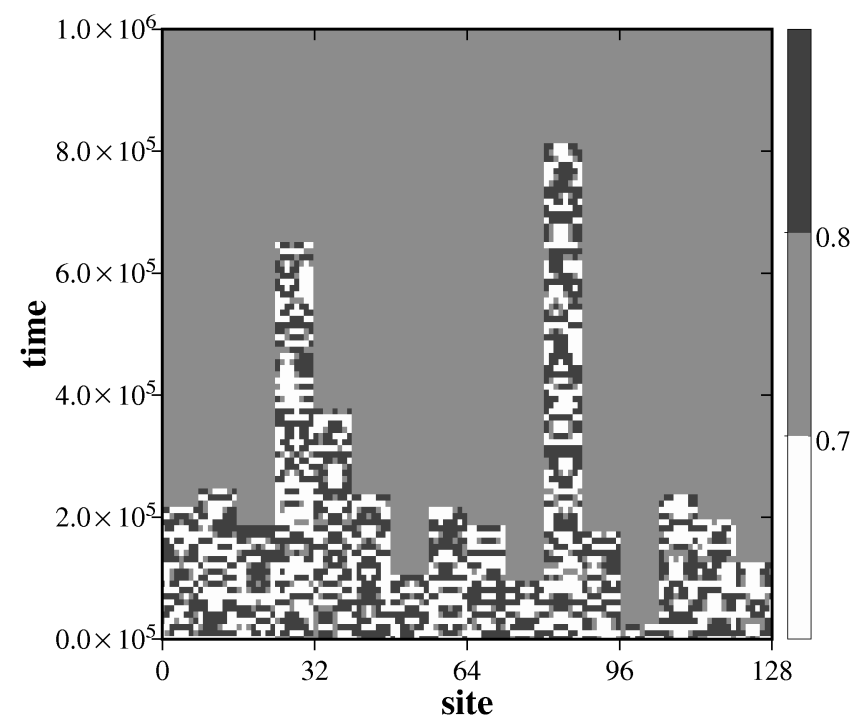

FIG. 4. Stabilizing uniform steady state: A large lattice ( $L=128)$ is controlled by an array of double pinning sites, placed at the boundaries of subdomains with length $L_{p}=8$. The state of the system was plotted at each 10000 th step. tained for the linear control scheme [obtained by linearizing (17)], which requires only the Jacobian to be known. In fact our computational resources were insufficient to observe even a single capture for $L>40$ with the linearized control. Generalizing this nonlinear approach to higher-dimensional systems remains a challenge.

To summarize, we have shown that the restrictions on the minimal density of periodically placed single pinning sites obtained by Qu and $\mathrm{Hu}$ [8] as a result of numerical simulations can in fact be obtained analytically from the stabilizability condition.

The efficiency of the control scheme can be improved significantly if one uses double pinnings instead of single ones. The homogeneous steady state becomes controllable for any values of the control parameters and the minimal density of pinning sites is reduced substantially. It is shown that the maximal distance between the pinnings depends on the strength of noise in the system and can be estimated analytically.

The appropriately chosen (using the LQ technique) feedback can decrease the capture time for the chaotic trajectory by enlarging the capture region. The introduction of nonlinearity into the control scheme can decrease this time even more significantly by effectively decoupling the large lattice into a number of smaller subdomains.

The authors thank J. C. Doyle for many fruitful discussions and J. Socolar and D. Egolf for valuable comments that led to improvements to the derivation of Eqs. (15) and (16). This work was partially supported by the NSF through Grant No. DMR-9013984. H. G. S. thanks C. Koch for the kind hospitality extended to him at Caltech and the Volkswagen Foundation for financial support.

[1] M. Ding et al., Phys. Rev. E 53, 4334 (1996); Y.C. Lai and C. Grebogi, Phys. Rev. E 50, 1894 (1994); J. Warncke, M. Bauer, and W. Martienssen, Europhys. Lett. 25, 323 (1994); D. Auerbach, Phys. Rev. Lett. 72, 1184 (1994).

[2] R. A. Katz, T. Galib, and J. Cembrola, J. Phys. IV (France) 4, 1063 (1994).

[3] A. Pentek, J. B. Kadtke, and Z. Toroczkai, Phys. Lett. A 224, 85 (1996).

[4] P. Colet, R. Roy, and K. Weisenfeld, Phys. Rev. E 50, 3453 (1994).

[5] V. Petrov, M. J. Crowley, and K. Showalter, Phys. Rev. Lett. 72, 2955 (1994).

[6] F. J. Romeiras et al., Physica (Amsterdam) 58D, 165 (1992); V. Petrov et al., Phys. Rev. E 51, 3988 (1995).

[7] K. Kaneko, Prog. Theor. Phys. 72, 480 (1984).

[8] G. Hu and Z. Qu, Phys. Rev. Lett. 72, 68 (1994).

[9] P. Dorato, C. Abdallah, and V. Cerrone, Linear-Quadratic Control: An Introduction (Prentice Hall, Englewood Cliffs, New Jersey, 1995).

[10] R. O. Grigoriev and M. C. Cross (to be published).

[11] E. Barreto and C. Grebogi, Phys. Rev. E 52, 3553 (1995).

[12] J. Kautsky, N. K. Nichols, and P. van Dooren, Int. J. Control 41, 1129 (1985).

[13] I. Aranson, D. Golomb, and H. Sompolinsky, Phys. Rev. Lett. 68, 3495 (1992). 